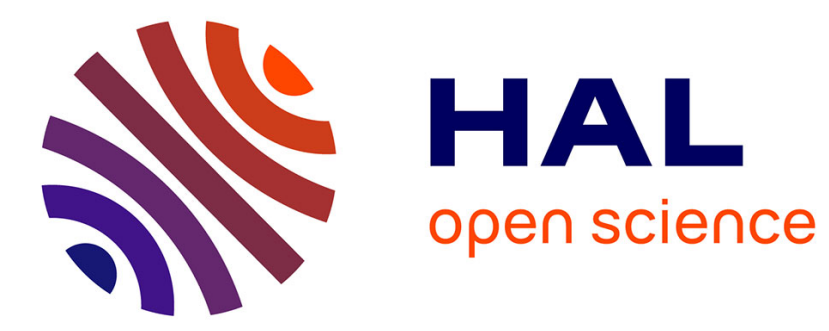

\title{
Multiple Wavelength Excitation 5-ALA Induced PpIX Fluorescence Spectroscopy in Guided Neurosurgery for Improving Glioma Classification
}

\author{
Arthur Gautheron, M Hébert, L Mahieu-Williame, J Guyotat, B Montcel
}

\section{- To cite this version:}

Arthur Gautheron, M Hébert, L Mahieu-Williame, J Guyotat, B Montcel. Multiple Wavelength Excitation 5-ALA Induced PpIX Fluorescence Spectroscopy in Guided Neurosurgery for Improving Glioma Classification. European Conferences on Biomedical Optics, 2021, SPIE, Jun 2021, Munich (virtual), Germany. 10.1117/12.2614590 . hal-03257711

HAL Id: hal-03257711

https://hal.science/hal-03257711

Submitted on 11 Jun 2021

HAL is a multi-disciplinary open access archive for the deposit and dissemination of scientific research documents, whether they are published or not. The documents may come from teaching and research institutions in France or abroad, or from public or private research centers.
L'archive ouverte pluridisciplinaire HAL, est destinée au dépôt et à la diffusion de documents scientifiques de niveau recherche, publiés ou non, émanant des établissements d'enseignement et de recherche français ou étrangers, des laboratoires publics ou privés. 


\title{
Multiple Wavelength Excitation 5-ALA Induced PpIX Fluorescence Spectroscopy in Guided Neurosurgery for Improving Glioma Classification
}

\author{
A. Gautheron ${ }^{a, *}$, M. Hébert ${ }^{b}$, L. Mahieu-Williame ${ }^{a}$, J. Guyotat $^{c}$, B. Montcel $^{a, *}$ \\ ${ }^{a}$ Univ Lyon, INSA-Lyon, Université Lyon 1, UJM-Saint Etienne, CNRS, Inserm, CREATIS UMR5220,U1206, \\ F-69616, Lyon, France \\ ${ }^{b}$ Univ Lyon, UJM-Saint-Etienne, CNRS, Institut d Optique Graduate School, Lab. Hubert Curien UMR5516, \\ F-42023, St Etienne, France \\ ${ }^{c}$ Hospices Civils de Lyon, Groupe Hospitalier Est, Service de Neurochirurgie, 59 Bvd Pinel, 69394,Lyon, Cedex, \\ France \\ *arthur.gautheron@creatis.insa-lyon.fr, bruno.montcel@creatis.insa-lyon.fr
}

\begin{abstract}
We present a methodology for PpIX parameters estimation based on multiwavelength excitation. This method aims to distinguish healthy tissues from tumour margins. It reduces the absolute estimation error of low density margins by $39.9 \%$. (C) 2021 The Author(s)
\end{abstract}

\section{Introduction}

Diffuse gliomas account for more than fifty percent of primitive brain tumors and are still hardly curable today. All subtypes of gliomas share the same highly infiltrative behavior of individual tumor cells. However surrounding infiltrated tissue often resemble normal tissues. The world health organization (WHO) classifies gliomas in 4 grades [1], but most studies commonly consider two separate groups having different biological, molecular and tissue properties: High Grade Gliomas (HGG) and Low Grade Gliomas (LGG). HGG are mainly malignant tumors (grades III and IV of WHO classification), while LGG are benign tumors (grades I and II). In both groups, infiltrative tumor cells are still difficult to identify during surgery. Pre-operative MRI combined with neuro-navigation is currently used to localize surgical tools and tumour cells in the operating theater but it shows strong limitations.

As a complementary method to pre-operative MRI, fluorescence microscopy has shown its relevance in neurooncology and 5-aminolevulonic acid (5-ALA) induced fluorescence of protoporphyrin IX (PpIX) is currently used through surgical microscopes [2]. However, its sensitivity is still limited when applied to low density infiltrative parts of High Grade Gliomas (HGG) or Low Grade Gliomas (LGG). To overcome sensitivity issues, various 5-ALA induce PpIX fluorescence spectroscopy methods have been proposed. Many studies on biopsies raised the presence of two states of PpIX with different fluorescence spectra, peaking at $634 \mathrm{~nm}$ and also at $620 \mathrm{~nm}$, in HGG as well as in LGG [3]. A recent 10 patient clinical trial demonstrated the presence of the second peak of fluorescence of PpIX at 620nm in non-extracted and freshly extracted living brain tissues, and its correlation with the pathological status of tissues [4].

The presence of the second peak of fluorescence of PpIX at $620 \mathrm{~nm}$ is known in solution and closely linked with the chemical microenvironment [5,6] or in cell culture. But its origin in vivo is still an open issue. Some works support the assumption that the origin of the peak at $620 \mathrm{~nm}$ in vivo is a different aggregate of PpIX. Other works [7] explained it by precursors of uroporphyrins or coproporphyrins. Furthermore, other endogenous fluorophores could play a important role in the fluorescence emission spectrum in the $600 \mathrm{~nm}$ region. The fitting of fluorescence 5-ALA induced fluorescence spectra in vivo is indeed multi-parametric. Then, there is a need to propose robust models to improve the accuracy of fitted biomarkers of PpIX fluorescence spectra.

In these works, we propose a new method using multiple wavelength excitation of the PpIX fluorescence. The model integrate the multiple excitation process and the estimation of contributions of both states without any a priori other endogenous fluorescence spectra. The results indicate that our method reduces the relative estimation error of the contributions of both states under $10 \%$ on average for each pathological category. 


\section{Material and methods}

\subsection{Multi excitation fluorescence model, estimation and error metrics}

The fluorescence emission spectrum used in this study relies on multiple excitation wavelengths. At each excitation wavelength $\lambda e$, the fluorescence spectrum $S_{\lambda_{e}}(\lambda)$ can be written :

$$
S_{\lambda e}(\lambda)=\alpha_{620} \cdot \eta_{620}\left(\lambda_{e}\right) \cdot S_{620}(\lambda)+\alpha_{634} \cdot \eta_{634}\left(\lambda_{e}\right) \cdot S_{634}(\lambda)+\varepsilon\left(\lambda_{e}, \lambda\right),
$$

where $S_{620}(\lambda)$, resp. $S_{634}(\lambda)$, are the normalized emission spectrum of PpIX $620 \mathrm{~nm}$, resp. $634 \mathrm{~nm} ; \eta_{620}$, resp. $\eta_{634}$, are the quantum yield at excitation wavelength of PpIX $620 \mathrm{~nm}$, resp. $634 \mathrm{~nm}, \alpha_{620}$, resp. $\alpha_{634}$, are the contribution at excitation wavelength of PpIX $620 \mathrm{~nm}$, resp. $634 \mathrm{~nm}$, and $\varepsilon\left(\lambda_{e}, \lambda\right)$ represents other endogenous fluorophores emission spectra.

Whether in the case of mono- or multi-excitation, we suggest to minimise the cost function :

$$
f\left(\alpha_{620}^{*}, \alpha_{634}^{*}\right)=w(\lambda) \cdot \sum_{\lambda e}\left(\alpha_{620}^{*} \cdot \eta_{620}\left(\lambda_{e}\right) \cdot S_{620}(\lambda)+\alpha_{634}^{*} \cdot \eta_{634}\left(\lambda_{e}\right) \cdot S_{634}(\lambda)-S_{\lambda e}(\lambda)\right)^{2},
$$

where $w(\lambda)$ is a user-defined weights function.

To compare estimation results $\alpha_{620}^{*}, \alpha_{634}^{*}$ with the ground truth $\alpha_{620}, \alpha_{634}$, errors were computed as :

$$
\begin{aligned}
\text { absolute estimation error : } E\left(\alpha_{620}^{*}, \alpha_{634}^{*}, \alpha_{620}, \alpha_{634}\right) & =\sqrt{\left(\alpha_{620}^{*}-\alpha_{620}\right)^{2}+\left(\alpha_{634}^{*}-\alpha_{634}\right)^{2}}, \\
\text { relative estimation error : } E_{\text {rel }}\left(\alpha_{620}^{*}, \alpha_{634}^{*}, \alpha_{620}, \alpha_{634}\right) & =\frac{E\left(\alpha_{620}^{*}, \alpha_{634}^{*}, \alpha_{620}, \alpha_{634}\right)}{\sqrt{\left(\alpha_{620}\right)^{2}+\left(\alpha_{634}\right)^{2}}} .
\end{aligned}
$$

\subsection{Digital Phantom}

In order to validate our method, we use a digital phantom which includes a parasitic fluorophore. The digital phantom is parameterized from experimental data obtained on liquid PpIX phantom made as in Ref. [6].

We consider two excitation wavelengths $\lambda e_{1}=385 \mathrm{~nm}$ and $\lambda e_{2}=405 \mathrm{~nm}$; quantum yield ratio for PpIX $620 \mathrm{~nm}$ $\eta_{\lambda_{e 2}} / \eta_{\lambda_{e 1}}=1.6176$; for PpIX $634 \mathrm{~nm} \eta_{\lambda_{e 1}} / \eta_{\lambda_{e 2}}=0.9743$, after Ref. [8]. For parasitic fluorophore, we decided to get values of lipofuscin : the quantum yield $\eta_{\lambda_{e 1}} / \eta_{\lambda_{e 2}}=0.75$ is used, after Ref. [9]; central emission wavelength $\lambda_{\text {para }} \approx 590 \mathrm{~nm}$ and standard deviation $\sigma_{\text {para }}=15 \mathrm{~nm}$ are used, after Ref. [10]. The parasitic fluorophore amplitude $A$ is set as 50\% of PpIX amplitude in healthy tissues : $A=363.2050$ a.u. as insipred by previous in vivo works [4]. In order to have a more realistic digital phantom, we decided to model acquisition noise. Therefore, we have a two-part noise : a Poisson noise $\mathscr{P}$ which mimics photon noise and an additive Gaussian white noise $\mathscr{N}$ mimics electronic noise.

This leads to the following function for spectrum generation :

$$
\begin{aligned}
S_{\lambda e}\left(\alpha_{620}, \alpha_{634}, \lambda\right) & =\left|\mathscr{P}\left[\alpha_{620} \cdot \eta_{620}\left(\lambda_{e}\right) \cdot S_{620}(\lambda)+\alpha_{634} \cdot \eta_{620}\left(\lambda_{e}\right) \cdot S_{634}(\lambda)+\varepsilon\left(\lambda_{e}, \lambda\right)\right]+\mathscr{N}\right|, \\
\text { where } \varepsilon\left(\lambda_{e}, \lambda\right) & =\eta\left(\lambda_{e}\right) \cdot \frac{A}{\sigma_{\text {para }} \sqrt{2 \pi}} e^{-\left(\frac{\lambda-\lambda_{\text {para }}}{\sqrt{2} \sigma_{\text {para }}}\right)^{2}} .
\end{aligned}
$$

\section{Results and discussion}

The previous study of Alston et al. [4] gives specific $\alpha_{620}, \alpha_{634}$ parameters for each one of the pathological statuses : solid HGG, margin HGG, low density HGG, LGG and Healthy. We take these values of $\alpha_{620}, \alpha_{634}$ parameters and Eq. 5 to generate fluorescence spectra associated to a specific pathological status. The weight function $w(\lambda)$ in Eq. 2 is zero except between $620 \mathrm{~nm}$ and $650 \mathrm{~nm}$, where it is equal to one.

We made a strict comparison between four different estimation methods. Alston et al. [4] estimation method, estimation based on excitation at $385 \mathrm{~nm}$ only, estimation based on excitation at $405 \mathrm{~nm}$ only, or excitation at both wavelengths. Results are shown in Fig 1.

In Fig. 1a, we notice that for the given digital phantom, the multi-excitation estimation method reduces at least by $41 \%$ the absolute estimation error (see eq. 3) compared to estimation at $385 \mathrm{~nm}$ wavelength only, by $23 \%$ compared to estimation at $405 \mathrm{~nm}$ wavelength only, and by $27 \%$ compared to Alston et al. [4] estimation for each pathological status. 

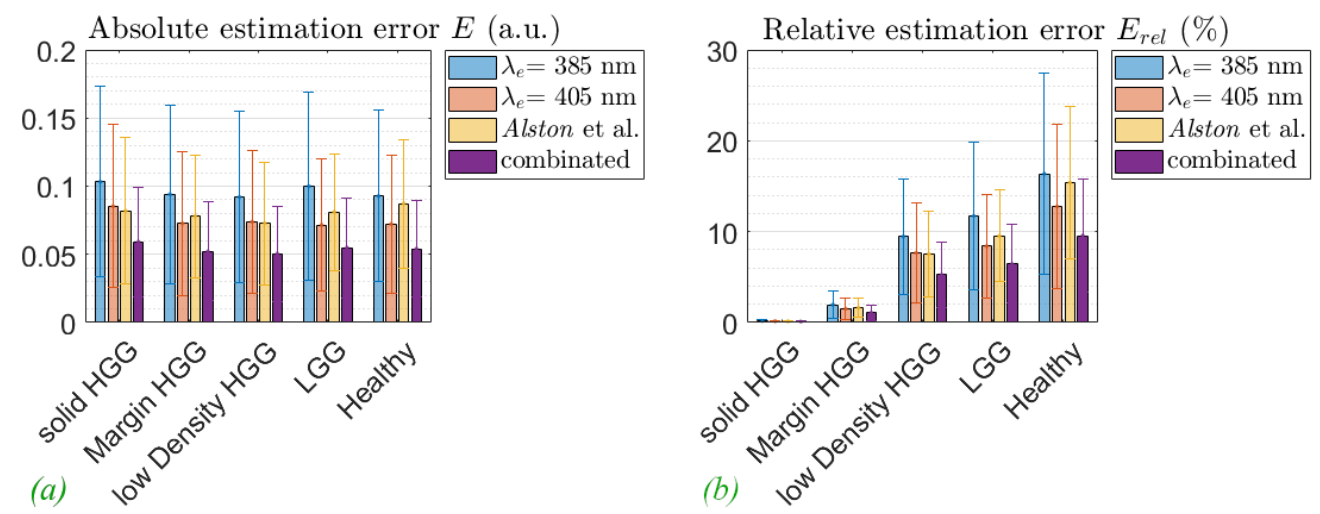

Fig. 1: (a) Absolute Estimation Error (see eq. 3) of $\alpha_{620}, \alpha_{634}$ by four different methods for each pathological status.

(b) Relative Estimation Error (see eq. 4) of $\alpha_{620}, \alpha_{634}$ by four different methods for each pathological status

In Fig. 1b, we notice that the highest relative estimation error (see eq. 4) is for the healthy tissue category. This seems to be a consequence of the small PpIX fluorescence intensity in a noisy environment whose signal to noise ratio at $630 \mathrm{~nm}$ equals 20,3 .

In conclusion, this work indicates that multi-wavelength excitation of the PpIX fluorophore with an estimation using all acquired spectra reduces the average relative estimation error, see eq. 3. In the case of two different excitation wavelengths, the average relative estimation error staid below $10 \%$ in all pathological status, whereas Alston et al. [4] relative estimation error staid only below $16 \%$ in average. More detailed results will be presented and discussed at the conference. This study confirms the usefulness of multi-wavelength excitation for distinguishing both states of PpIX.

Acknowledgments: This work has been funded by LABEX PRIMES (ANR-11-LABX-0063) of Université de Lyon, within the program "Investissements d'Avenir" (ANR-11-IDEX-0007) operated by the French National Research Agency (ANR) and carried out within the framework of France Life Imaging (ANR-11-INBS-0006). We also want to acknowledge the PILoT facility for the support provided on data acquisition.

\section{References}

1. D. N. Louis et al. The 2016 World Health Organization Classification of Tumors of the Central Nervous System: a summary. Acta Neuropathol., 131(6):803-820, Jun 2016.

2. W. Stummer et al. Technical principles for protoporphyrin-IX-fluorescence guided microsurgical resection of malignant glioma tissue. Acta Neurochir., 140(10):995-1000, 1998.

3. B. Montcel et al. Two-peaked 5-ALA-induced PpIX fluorescence emission spectrum distinguishes glioblastomas from low grade gliomas and infiltrative component of glioblastomas. Biomed. Opt. Express, 4(4):548-558, Apr 2013.

4. L. Alston et al. Spectral complexity of 5-ALA induced PpIX fluorescence in guided surgery: a clinical study towards the discrimination of healthy tissue and margin boundaries in high and low grade gliomas. Biomed. Opt. Express, 10(5):2478-2492, May 2019.

5. T. B. Melø et al. The physicochemical state of protoporphyrin IX in aqueous solution investigated by fluorescence and light scattering. Biophys. Chem., 25(1):99-104, Nov 1986.

6. L. Alston et al. Nonlinear relation between concentration and fluorescence emission of protoporphyrin IX in calibrated phantoms. J. Biomed. Opt., 23(9):097002, Sep 2018.

7. M. Zanello et al. Multimodal optical analysis discriminates freshly extracted human sample of gliomas, metastases and meningiomas from their appropriate controls. Sci. Rep., 7:41724, Feb 2017.

8. C. Jonin et al. Two photon excited fluorescence and hyper rayleigh scattering of protoporphyrin ix. Journal of Photochemistry and Photobiology A: Chemistry, 402:112812, 2020.

9. J. Wassell et al. A role for vitamin A in the formation of ocular lipofuscin. Br. J. Ophthalmol., 81(10):911918, Oct 1997.

10. A. C. Croce et al. Autofluorescence Spectroscopy and Imaging: A Tool for Biomedical Research and Diagnosis. Eur. J. Histochem., 58(4), Oct 2014. 